\title{
Dependability for high-tech systems: an industry-as-laboratory approach*
}

\author{
Ed Brinksma \\ Embedded Systems Institute, Eindhoven \\ University of Twente, Enschede \\ The Netherlands \\ ed.brinksma@esi.nl
}

\author{
Jozef Hooman \\ Embedded Systems Institute, Eindhoven \\ Radboud University Nijmegen \\ The Netherlands \\ jozef.hooman@esi.nl
}

\begin{abstract}
The dependability of high-volume embedded systems, such a consumer electronic devices, is threatened by a combination of quickly increasing complexity, decreasing time-to-market, and strong cost constraints. This poses challenging research questions that are investigated in the Trader project, following the industry-as-lab approach. We present the main vision of this project, which is based on a model-based control paradigm, and the current status of the project results.
\end{abstract}

\section{Introduction}

High-tech systems by definition are constructed using cutting edge technology, and consequently embedded system technology plays a major and often even decisive role in such systems, whether they are mobile phones, HDTVs, medical equipment, cars, airplanes, etc. The integration of embedded hardware and software into larger systems has lifted the issue of dependability of the embedded components to the level of the embedding high-tech system. At that level the integral system dependability is not only affected by the dependability of its individual components, but mainly an emergent quality of the interactions between these components and the system environment. Controlling the complexity of these interactions is one of the major challenges of high-tech system design, and the presence dependability problems in almost all application domains is a well-documented fact of life.

In this paper we want to report on a model-based approach to system dependability. Although model-based design has already been advocated for a considerable time by

\footnotetext{
*This work has been carried out as part of the Trader project under the responsibility of the Embedded Systems Institute. This project is partially supported by the Dutch Ministry of Economic Affairs under the Bsik program.
}

(mainly) the academic community as a way forward to address complex embedded systems engineering tasks, the industrial uptake is lagging behind. To ensure the practical relevance of the research, it is being carried out following an industry-as-laboratory approach [5]. This means that concrete cases are studied in their industrial context to promote the applicability and scalability of solution strategies under the relevant practical constraints.

The concrete case that we discuss is based on the collaborative research project Trader of the Embedded Systems Institute (ESI) with NXP and other academic and industrial partners on system dependability for high-volume systems. High-volume systems are characterized by the fact that because of their production in large quantities, the cost per item should be (very) low. This seriously restricts the possibility to address dependability by classical means, such as over-dimensioning of critical components. One of the main ideas in the project is to use concepts from model-based control to achieve dependability.

The rest of the paper is organized as follows: Sect. 2 contains an outline of the project, Sect. 3 outlines the modelbased philosophy of the project, and Sect. 4 reports of the current status of the results. We draw our conclusions and list some future work in Sect. 5 .

\section{Outline of the Trader Project}

In the Trader project, academic and industrial partners collaborate to optimize the dependability of high-volume products, such as consumer electronic devices. The project partners involved are: NXP Semiconductors, NXP Research, ESI, TASS, IMEC (Belgium), Twente University, the Technical University of Delft, the University of Leiden, and the Design Technology Institute (DTI) at the Eindhoven University of Technology. The project started September 2004, with a duration of five years, and includes seven $\mathrm{PhD}$ students and two postdocs. The so-called Carrying Industrial Partner (CIP) of this project is NXP Semiconductors, providing the project with a focus on multimedia products. 
NXP has provided the problem statement and proposes relevant case studies, which in the case of Trader are taken from the TV domain. The problem statement is based on the observation that the combination of increasing complexity of consumer electronic products and decreasing time-tomarket will make it extremely difficult to produce totally reliable devices that meet the dependability expectations of customers.

A current high-end TV is already a very complex device which can receive analog and digital input from many possible sources and using many different coding standards. It can be connected to various types of recording devices and includes many features such a picture-in-picture, teletext, sleep timer, child lock, TV ratings, emergency alerts, TV guide, and advanced image processing. Moreover, there is a growing demand for features that shared with other domains, such as photo browsing, MP3 playing, USB, games, databases, and networking. As a consequence, the amount of software in TVs has seen an exponential increase from 1 $\mathrm{KB}$ in 1980 to more than $20 \mathrm{MB}$ in current high-end TVs.

Also the hardware complexity is increasing rapidly, for instance for the support of real-time decoding and processing of high-definition images for large screens and multiple tuners. To meet the hard real-time requirements a TV is designed as a system-on-chip with multiple processors, various types of memory, and dedicated hardware accelerators.

At the same time, there is a strong pressure to decrease time-to-market. To be able to realize products with many new features quickly, components developed by others have to be incorporated. This includes so-called third-party components, e.g., for audio and video standards. Moreover, there is a clear trend towards the use of downloadable components to increase product flexibility and to allow new business opportunities (selling new features, games, etc.). Given the large number of possible user settings and types of input, exhaustive testing is impossible. Also, the product must be able to tolerate certain faults in the input. Customers expect, for instance, that products can cope with deviations from coding standards or bad image quality.

Although companies invest a lot of attention and effort to avoid faults in released products, it is expected that without additional measures both internal and external faults are serious threats to product dependability. The cost of non-quality, however, is high, because it leads to many returned products, it damages brand image, and reduces market share.

The main goal of the Trader project is to improve the user-perceived dependability of high-volume products. The aim is to develop techniques that can compensate and mask faults in released products, such that they satisfy user expectations. The main challenge is to realize this without increasing development time and, given the domain of highvolume products, with minimal additional hardware costs and ithout degrading performance. Hence, classical faulttolerance techniques that rely a lot on additional redundancy and resources (e.g., duplication or even triplication of hardware and software) are not suitable in this domain.

In our presentation the terminology of [1]is adopted. A failure of a system with respect to an external specification is an event that occurs when a state change leads to a run that no longer satisfies the external specification. An error is the part of the system state that may lead to a failure. For instance, a wrong memory value or a wrong message in a queue. A fault is the adjudged or hypothesized cause of an error which is not part of the system state. Examples of faults are programming mistakes (e.g., divide by zero) or unexpected input.

\section{Model-Based Approach}

Looking at a number of failures of consumer electronic devices, it is often the case that a user can immediately observe that something is wrong, whereas the system itself is completely unaware of the problem. Systems are often realized in a way that corresponds to the open-loop approach in control theory; for a certain input, the required actions are executed, but it is never checked whether these actions have the desired effect on the system and whether the system is still in a healthy state.

The main approach of the Trader project is to "close the loop" and to add a kind of feedback control to products. By monitoring the system and comparing system observations with a model of the desired behaviour at run-time, the system gets a form of run-time awareness which makes it possible to detect that its customer-perceived behavior is (or is likely to become) erroneous. In addition, the aim is to provide the system with a strategy to correct itself.

The main ingredients of such a run-time awareness and correction approach are depicted in Fig. 1.

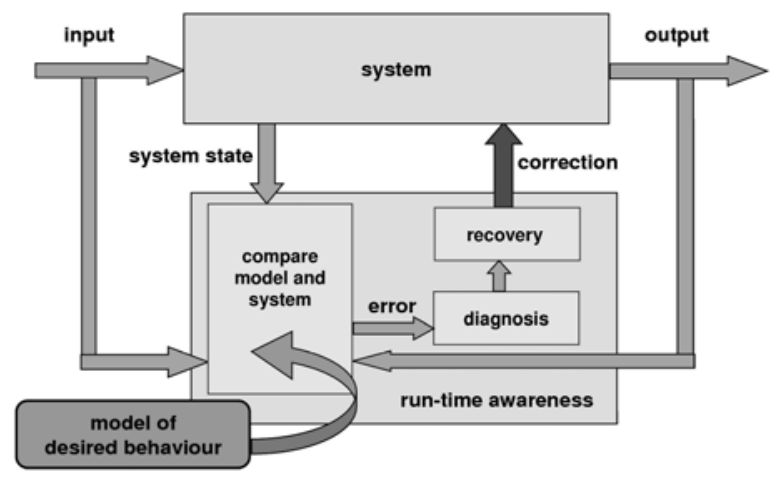

Figure 1. Adding awareness at run-time

We discuss the main parts, giving examples from the TV domain: 
- Observation: observe relevant inputs, outputs and internal system states. For instance, for a TV we may want to observe keys presses from the remote control, internal modes of components (dual/single screen, menu, mute/unmute, etc), load of processors and busses, buffers, function calls to audio/video output, sound level, etc.

- Error detection: detect errors, based on observations of the system and a model of the desired system behaviour.

- Diagnosis: in case of an error, find the most likely cause of the error.

- Recovery: correct erroneous behaviour, based on the diagnosis results and information about the expected impact on the user.

Important part of the approach depicted in Fig. 1 is the use of models at run-time. Note that for complex systems it will be infeasible to include a complete model of desired system behaviour, but the approach allows the use of partial models, concentrating on what is most relevant for the user. Moreover, we can apply this approach hierarchically and incrementally to parts of the system, e.g., to third-party components. Typically, there will be several awareness monitors in a complex systems, for different components, different aspects, and different kinds of faults.

The analogy between self-controlling software and control theory has already been observed in [10]. Garlan et al [9] have developed an adaptation framework where system monitoring might invoke architectural changes. Using performance monitoring, this framework has been applied to the self-repair of web-based client-server systems. Related work that also takes cost limitations into account can be found in the research on fault-tolerance of largescale embedded systems [13]. They apply the autonomic computing paradigm to systems with many processors to obtain a healing network, also using a kind of controllerplant feedback loop. Related work on adding a control loop to an existing system is described in the middleware approach of [14] where components are coupled via a publishsubscribe mechanism.

\section{Current Status of Trader}

In this section, we give a brief description of the research activities and the current status of the Trader project. First we discuss the research on the ingredients of the global awareness vision depicted in Fig. 1: observation, (Sect. 4.1), modeling system behaviour (Sect. 4.2), error detection (Sect. 4.3), diagnosis (Sect. 4.4), and recovery (Sect. 4.5). Finally, we mention research on reliability improvements during development and user perception in Sect. 4.7 and Sect. 4.6, respectively.

\subsection{Observation}

To observe relevant aspects of the system, both hardware and software techniques are investigated. Hardware-related work in Trader currently aims at exploiting mechanisms already available in hardware, such as the on-chip debug and trace infrastructure, to monitor values for range checking, call stacks (functions, parameters, and result values), and memory arbiters. The observation of software behaviour is mainly done by code instrumentation using aspect-oriented techniques, partly based on results from ESI-project Ideals project $[6,7]$. A specialized aspect-oriented framework called AspectKoala [19] has been developed on top of the component model Koala which is used at NXP.

\subsection{Modeling Desired System Behaviour}

Important part of the model-based approach described in Sect. 3 is the use of a model of desired system behaviour at run-time. Experience in Trader and other ESI projects indicates that such models are usually not available in industry and that it is often difficult to obtain such models. In industrial practice, system requirements are usually distributed over many documents and databases. Hence, part of the ESI research in Trader explicitly addresses the construction of a high-level system model.

Since the TV domain is the main source of case studies in Trader, we have developed a high-level model of a TV from the viewpoint of the user. It captures the relation between user input, via the remote control, and output, via images on the screen and sound. A few first experiments indicated that the use of state machines leads to suitable models for the control behaviour of the TV. But it also revealed that it was very easy to make modeling errors, for instance, because there are many interactions between features. Examples are relations between dual screen, teletext and various types of on-screen displays that remove or suppress each other.

To allow quick feedback on the user-perceived behaviour and to increase the confidence in the fidelity of the model, Matlab/Simulink [11] is used to obtain executable models. Stateflow is exploited for the control part, whereas the streaming part of a TV is modeled by means of the Image and Video Processing toolbox of Simulink. External events can be generated by clicking on a picture of a remote control. Output is visualized by means of Matlab's video player and a scope for the volume level. This visualization of the user view on input and output of the model turned out to be very useful to detect modeling errors and undesired feature interactions. In addition, we investigate the possibilities of 
formal model-checking and test scripts to improve model quality.

\subsection{Error Detection}

Various techniques for error detection are investigated such as hardware-based deadlock detection and range checking. An approach which checks the consistency of internal modes of components turned out to be successful to detect teletext problems due to a loss of synchronization between components [17].

To enable quick experimentation with model-based error detection, we have developed a framework which allows the use of models at run-time. The framework has been implemented on top of Linux, to comply with the trend towards open-source software and the use of Linux in TVs. In the framework, one can include a particular System Under Observation (SUO) and a specification model of the desired system behaviour. The design of the awareness framework is shown in Fig. 2. The SUO and the awareness monitor are

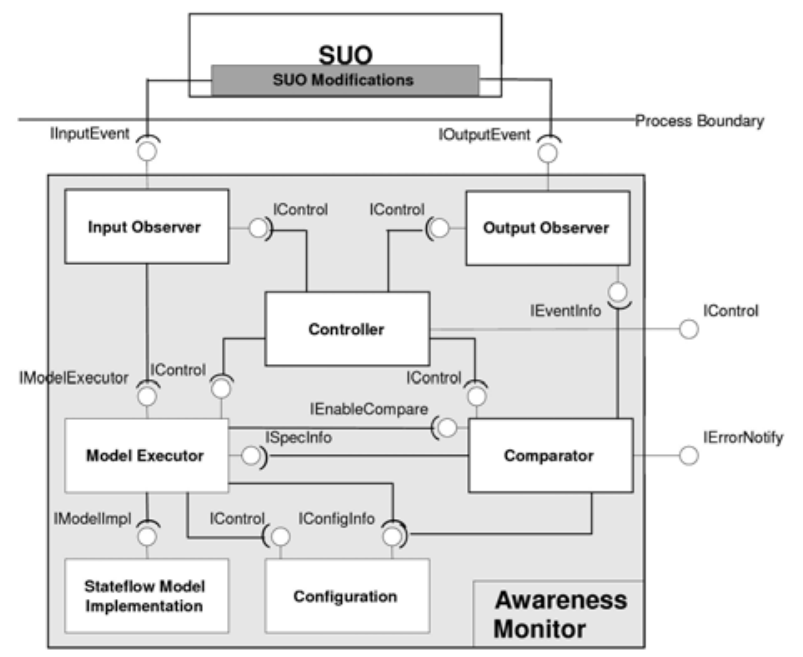

Figure 2. Design of the awareness framework

separate processes and Unix domain sockets are used for inter-process communication. The SUO has to be adapted slightly, to send messages with relevant input and output events (which may also include internal states) to Input and Output Observers. An executable specification model of the SUO in Stateflow can be included by using the code generation possibilities of Stateflow. The generated C-code can be included easily, allowing quick experiments with different models. It is executed using the Model Executer component, based on event notifications from the Input Observer. Information about relevant input and output events is stored in the Configuration component. The Comparator component compares relevant model output with system output which is obtained from the Output Observer. The Controller initiates and controls all components, except for the Configuration component which is controlled by the Model Executor.

Experiments with earlier versions of the framework indicated that the Comparator should not be too eager to report errors; small delays in system-internal communication might easily lead to differences during a short time interval. Hence, in the current framework the user of the framework can specify, for each observable value: (1) a threshold for the allowed maximal deviation between specification model and system, and (2) a maximum for the number of consecutive deviations that are allowed before an error will be reported.

Another relevant parameter is the frequency with which time-based comparison takes place. This can be combined with event-based comparison by specifying in the specification model when comparison should take place and when not (e.g., when the system is in an unstable state between certain modes). Observe that we have to make a trade-off between taking more time to avoid false errors and reporting errors fast to allow quick repair.

Related to our approach is a method to wrap COTS components and monitor them using specifications expressed as a UML state diagrams is presented in [16]. Other related work consists of assertion-based approaches such as runtime verification [4]. For instance, monitor-oriented programming [3] supports run-time monitoring by integrating specifications in the program via logical annotations. In our approach, we aim at minimal adaptation of the software of the system, to be able to deal with third-party software and legacy code. Moreover, we also monitor real-time properties, which are not addressed by the techniques cited above. Closely related in this respect is the MaC-RT system [15] which also detects timeliness violations. Main difference with our approach is the use of a timed version of Linear Temporal Logic to express requirements specifications, whereas we use executable timed state machines to promote industrial acceptance and validation.

\subsection{Diagnosis}

The diagnoses techniques developed within Trader are based on so-called program spectra [20]. The approach has already been applied to a few examples in the TV domain. As an illustration, we describe one of the first experiments on TV software in which a teletext error has been injected. First the $\mathrm{C}$ code is instrumented to record which blocks are executed. In the example there were 60000 blocks. Next, for each sequence of key presses, a so-called scenario, for each block it is recorded whether it has been executed or not between two key presses. This leads to a vector, a socalled spectrum, for each block. In our example it turns out 
that during a scenario of 27 key presses 13796 blocks were executed. Moreover, based on some error detection mechanism, it is recorded for each key press whether it leads to error or not. In the example, this leads to an error vectors of length 27 . Next, the similarity between the error vector and the spectra is computed. Finally, the blocks are ranked according their similarity. In the particular experiment with the teletext error, the block which contains the fault appeared on the first place in the ranking. Also in other case studies the application results of this technique are encouraging.

\subsection{Recovery}

Part of the recovery research concentrates on load balancing. Project partner IMEC has demonstrated the possibility to migrate an image processing task from one processor to another, which leads to improved image quality in case of overload situations (e.g., due to intensive error correction on a bad input signal). NXP Research investigates the possibility to make memory arbitration more flexible such that it can be adapted at run-time to deal with problems concerning memory access. At Twente University, a framework for partial recovery has been developed which allows independent recovery of parts of the system, the so-called recoverable units. The framework includes a communication manager, which controls the communication between recoverable units, and a recovery manager, which executes the recovery actions such as killing and restarting units. To realize these concepts, a reusable fault tolerance library has been implemented. A few first experiments in the multimedia domain show that after some refactoring of the system, independent recovery of parts of the system is possible without large overhead.

\subsection{User Perception}

The user perception of reliability is addressed by project partner DTI. The aim is to capture user-perceived failure severity, to get an indication of the level of user-irritation caused by a product failure. By means of controlled experiments with TV users, the impact of characteristics such as product usage, user group, and function importance is investigated. During experiments, it turned out that also failure attribution has a significant impact. For instance, users, when asked, rank both image quality and a motorized swivel, which can be used to turn the TV, as important. Under observation, however, users often turn out to be very tolerant concerning bad image quality (which is attributed to external sources), but get irritated if the swivel doesnot work correctly.

\subsection{Improvements During Development}

Part of the Trader research is also related to dependability improvements during development. This includes the use of code analysis to prioritize the warnings of a software inspection tool such as QA-C [2] and reliability analysis at the architectural level [18]. The stress testing approach of TASS artificially takes away shared resources, such as CPU or bus bandwidth, to simulate the occurrence of errors or the addition of an additional resource user. The study of the effect of such overload situations on the system behaviour and its fault-tolerant mechanisms has shown to be very useful in the TV domain. A so-called CPU eater, which consumes CPU cycles at the application level in software, is already included in the current development software and can be activated by system testers.

\section{Conclusion and future Work}

Although the Trader project has still some time to go, it is already clear that its particular model-based approach to system dependability is very promising. The use of models as system components to give the system a certain capacity to monitor and correct its behaviour, implements ideas from feedback control at the level of integrated systems. It constitutes a paradigm switch from the best-effort, open-loop approach that is traditional in software-related design, to the closed-loop control-based approach. The latter is much more suitable for the reality of high-tech systems in which errors are unavoidable emergent features of the system complexity.

The concept of model-based system level control is also quite flexible, in the sense that one can vary between lightweight models with limited corrective capacities, and more elaborate models with stronger feedback mechanisms. In the high-volume context, the constraint to minimize overhead is limiting factor. Certainly, much more research will be needed to obtain a more complete understanding of the potential and limitations of this approach in the a priori vast range of different application domains.

The choice for an industry-as-laboratory format for the Trader project has helped a lot in focussing on techniques and approaches that have a high potential for being absorbed by industry. Already now, some of the intermediate results have found their way into industry. We firmly believe in the potential of this research format to achieve a productive combination between real research and innovation.

Future activities in the Trader project will address further development of the awareness framework. Our Linux-based awareness framework, has been validated by means of model-to-model experiments. That is, we have compared a specification model with code generated from models of the 
SUO. Currently, the framework is used for awareness experiments with the open source media player MPlayer [12], investigating both correctness and performance issues. Next our approach will be applied in the TV domain at NXP, following the industry-as-lab paradigm. Important topic of research concerns the optimal integration of various techniques for observation, error detection, diagnosis, and recovery.

In parallel, the model-based run-time awareness concept is also exploited in the domain of printer/copiers at the company Océ in the context of the ESI-project Octopus [8], which started recently.

\section{References}

[1] A. Avizienis, J.-C. Laprie, B. Randell, and C. Landwehr. Basic concepts and taxonomy of dependable and secure computing. IEEE Transactions on Dependable and Secure Computing, 1(1):11-33, 2004.

[2] C. Boogerd and L. Moonen. Prioritizing software inspection results using static profiling. In SCAM '06: Proc. Workshop on Source Code Analysis and Manipulation, pages 149-160. IEEE Computer Society, 2006.

[3] F. Chen, M. D'Amorim, and G. Rosu. A formal monitoringbased framework for software development and analysis. In Proceedings ICFEM 2004, volume 3308 of LNCS, pages 357-372. Springer-Verlag, 2004.

[4] S. Colin and L. Mariani. Run-time verification. In Proceedings Model-Based Testing of Reactive Systems, volume 3472 of LNCS, pages 525-555. Springer-Verlag, 2005.

[5] C.Potts. Software-engineering research revisited. IEEE Software, 19(9):19-28, 1993.

[6] P. Durr, G. Gülesir, L. Bergmans, M. Aksit, and R. van Engelen. Applying AOP in an industrial context: An experience paper. In Proc. Workshop on Best Practices in Applying Aspect-oriented Software Development. AspectOriented Software Association, 2006.

[7] Embedded Systems Institute. The Ideals project, 2007. http://www.esi.nl/ideals/.

[8] Embedded Systems Institute. The Octopus project, 2007. http://www.esi.nl/octopus/.

[9] D. Garlan, S. Cheng, and B. Schmerl. Increasing system dependability through architecture-based self-repair. In Architecting Dependable Systems, volume 2677 of LNCS, pages 61-89. Springer-Verlag, 2003.

[10] M. M. Kokar, K. Baclawski, and Y. A. Eracar. Control theory-based foundations of self-controlling software. IEEE Intelligent Software, pages 37-45, 1999.

[11] The Mathworks. Matlab/Simulink, 2007. http://www. mathworks.com/.

[12] MPlayer. Open source media player, 2007. http://www.mplayerhq.hu/.

[13] S. Neema, T. Bapty, S. Shetty, and S. Nordstrom. Autonomic fault mitigation in embedded systems. Engineering Applications of Artificial Intelligence, 17:711-725, 2004.

[14] J. Parekh, G. Kaiser, P. Gross, and G. Valetto. Retrofitting autonomic capabilities onto legacy systems. Cluster Computing, 9(2):141-159, 2006.
[15] U. Sammapun, I. Lee, and O. Sokolsky. Checking correctness at runtime using real-time Java. In Proc. 3rd Workshop on Java Technologies for Real-time and Embedded Systems (JTRES'05), 2005.

[16] M. E. Shin and F. Paniagua. Self-management of COTS component-based systems using wrappers. In Computer Software and Applications Conference (COMPSAC 2006), pages 33-36. IEEE Computer Society, 2006.

[17] H. Sözer, C. Hofmann, B. Tekinerdogan, and M. Aksit. Detecting mode inconsistencies in component-based embedded software. In DSN Workshop on Architecting Dependable Systems, 2007.

[18] H. Sözer, B. Tekinerdogan, and M. Aksit. Extending failure modes and effects analysis approach for reliability analysis at the software architecture design level. In Architecting Dependable Systems IV, volume 4615 of LNCS, pages 409433. Springer-Verlag, 2007.

[19] P. van de Laar and R. Golsteijn. User-controlled reflection on join points. Journal of Software, 2(3):1-8, 2007.

[20] P. Zoeteweij, R. Abreu, R. Golsteijn, and A. van Gemund. Diagnosis of embedded software using program spectra. In Proc. 14th Conference and Workshop on the Engineering of Computer Based Systems (ECBS'07), pages 213-220, 2007. 